Vacuum Brake.-The engineers of the French Northern Railway have made satisfactory experiments with the vacuum brake. MM. Sartiaux and Lartique have devised some ingenious arrangements for bringing it into automatic action, if any mistake is made respecting the crossings. Distressed passengers, who need help, can operate the brake instead of ringing a bell.-Nature.

C.

An African Sea.-A writer in the Scientific American has lately estimated the amount of evaporation from the surface of the proposed Algerian Sea, the reduction of the ocean level, and the time required to fill the sea with a bed of salt. In his calculations he seems to have omitted any consideration of the amount of vapor that would be recondensed, over the sea or in the neighborhood of its banks, so as to find its way back into the reservoir from which it was drawn. The elements involved in the question are too numerous to be determined by a few ingenious numerical calculations, and the discussions in the French Academy show that there is no lack of ahle engineers and physicists, who are thoroughly competent and willing to look at the project in all its climatic and economical bearings, before any final decision is made.-Comptes Rendus.

C.

\title{
Recomposition at Temperatures Higher than those of
} Dissociation.-It is well known that most bodies are decomposed under the influence of heat, and that if the temperature is high enough, their decomposition is complete. It seems natural to suppose, that above this temperature the compounds could not exist. But we have demonstrated, by the study of many compounds of silicium, that this conclusion is too general. 1 . The sesquichloride of silicium, in particular, which is very stable at ordinary temperatures, begins to decompose at about $350^{\circ} \mathrm{C}$.; its decomposition is complete at about $800^{\circ}$. It may be represented by the formula, $2 \mathrm{Si}_{2} \mathrm{Cl}_{3}=3 \mathrm{Si} \mathrm{Cl}_{2}+\mathrm{Si}$. The sesquichloride is formed again, if we place the products of its decomposition in a porcelain tube, at about $1200^{\circ}: 3 \mathrm{Si} \mathrm{Cl}_{2}+\mathrm{Si}=2 \mathrm{Si}_{2} \mathrm{Cl}_{3}$. The sesquichloride thus formed may be isolated by rapid cooling. If it is brought gradually into parts of the tube where the temperature does not exceed $800^{\circ}$, it is decomposed, and gives crystals of silicium which obstruct the tube. In this case we get bichloride, which boils at $58^{\circ}$, instead of sesquichloride, boiling at $146^{\circ}$. The sesquichloride of silicium presents, therefore, a great stability at a temperature much higher, as well as at a temperature much lower, than that which determines its 